\title{
Study of the behaviour of lysozyme oligomers in solutions by the molecular dynamics method at different temperatures
}

\author{
lu. V. Kordonskaya ${ }^{1,2}$, V. I. Timofeev1, 2, lu. A. Dyakova1, 2, M. A. Marchenkova², lu. V. Pisarevskiy ${ }^{1,2}$, \\ M. V. Kovalchuk ${ }^{1,2}$

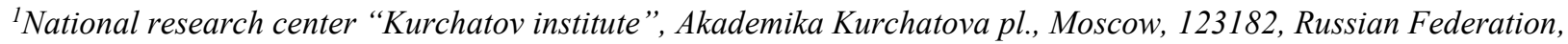 \\ ${ }^{2}$ Shubnikov Institute of Crystallography of Federal Scientific Research Centre "Crystallography and Photonics" of Russian Academy \\ of Sciences, 59, Leninsky Prospekt, Moscow, 119333, Russian Federation \\ yukord@mail.ru
}

Using the molecular dynamics simulation method, the stability of lysozyme octamer and two types of dimer (A and B) formed in solution under conditions of crystallization of tetragonal syngony was studied. In order to investigate the influence of $\mathrm{NaCl}$ precipitant ions bound to the protein in the crystal, various combinations of sodium and chloride ions associated with lysozyme molecule were probed: 1) with $\mathrm{Na}$ and $\mathrm{Cl}$ ions, 2) only with $\mathrm{Na}$ ions, and 3) without any ions. Using the GROMACS program, 100-ns molecular dynamics trajectories of the oligomers in the presence and absence of precipitant in water were calculated at different temperatures from 278 to $318 \mathrm{~K}$.

To evaluate the stability of oligomers, RMSF (Root Mean Square Fluctuations) graphs were plotted at every simulated temperature.

As a result, flexibilities of octamer and dimer A have regularly increased with the temperature growth only in the case of considering precipitant ions embedded in the crystal structure. The RMSF values of dimer B are approximately the same at temperatures from 283 to $313 \mathrm{~K}$ and become higher at $318 \mathrm{~K}$ for all simulations whether they were performed with bound precipitant ions or not.

Thus, the importance of $\mathrm{Na}$ and $\mathrm{Cl}$ ions associated with the lysozyme is shown as only results of simulating oligomer models containing precipitant ions are consistent with the ones obtained by small-angle x-ray scattering experiments on crystallization lysozyme solutions [1-2].

[1] Kovalchuk, M. V., Blagov, A.E., Dyakova, Y.A., Gruzinov, A.Y., Marchenkova, M.A., Peters, G.S., Pisarevsky, Y. V., Timofeev, V.I., Volkov, V. V. (2016). Crystal Growth \& Design 16 (4), 1792.

[2] Boikova, A. S., Dyakova, Y. A., Ilina, K. B., Konarev, P. V., Kryukova, A. E., Kuklin, A. I., Marchenkova, M. A., Nabatov, B. V., Blagov, A. E., Pisarevsky, Y. V. \& Kovalchuk, M. V. (2017). Acta Cryst. D73, pp. 591-599

\section{Keywords: molecular dynamics; protein crystal growth}

This study was supported in part by the Ministry of Science and Higher Education within the State assignment FSRC "Crystallography and Photonics» RAS and by the Russian Foundation for Basic Research (project number 19-29-12042 mk) and by the NRC “Kurchatov Institute” (№ 1360).

This work has been carried out using computing resources of the federal collective usage center Complex for Simulation and Data Processing for Mega-science Facilities at NRC “Kurchatov Institute”, http://ckp.nrcki.ru/ 